\title{
Grey Relational Analysis on Disposable Income and Consumption Expenditure for Urban Residents
}

\author{
Kuang Wang \\ Basic Teaching and Research Institute, Bohai University, Jinzhou, 121013, China \\ xiaowangkuang@126.com
}

Keywords: disposable income of residents; consumption expenditure; grey relational analysis

\begin{abstract}
The important task of China's current economic work is to stimulate domestic demand and stimulate consumption. The government needs to adjust its income distribution policy and use income adjustment measures to expand internal consumption demand. Under this macrobackground, this paper carries out research on the disposable income and consumption expenditure data of urban residents from 2013 to 2016, which has important theoretical and practical significance. Firstly, the mathematical model of grey relational analysis is constructed. Then, the disposable income index and the household consumption expenditure index are explained. Finally, the steps of solving the data mean, calculating the correlation coefficient, calculating the correlation degree, the rank of relevance degree are grayed out and illustrate the analysis results. The research results in this paper can provide theoretical basis and scientific methods for government departments to formulate policies.
\end{abstract}

\section{Introduction}

Grey correlation analysis, based on the similarity or dissimilarity of the development trends among factors, measures a degree of correlation between factors, overcomes the shortcomings of regression analysis and other methods in mathematical statistics, and does not pursue large sample size, and does not require the sequence of analysis obeying a typical probability distribution, a small amount of calculation and a simple calculation process. Disposable income of residents, which refers to the sum of residents' final consumption expenditures and savings, that is, the income that residents can use for discretion. It includes both cash income and entity income. Consumption of residents, refers to the total expenditure of residents used to meet the daily consumption needs of the family, including both cash consumption expenditure and entity consumption expenditure. This paper uses the grey correlation analysis method to quantitatively study the degree of influence from the perspective of the linkage development between disposable income of residents and consumption expenditures, overcomes the relative deficiencies of traditional research methods and accurately grasps the disposable income of residents and consumption expenditure of residents. The degree of relevance provides theoretical basis and scientific methods for government agencies to formulate policies, increases the disposable income of residents, and guides residents to rational consumption.

\section{Model on Grey Relational Analysis}

The basic idea of grey relational analysis is to judge whether the connection is close or not according to the similarity of the geometry of the sequence curve. The closer the curve is, the greater the degree of association between the corresponding sequences, and vice versa. The greater the grey correlation, the more consistent the two factors change. This paper builds a mathematical model according to the solution steps.

Step 1, Data normalization. Use the average of all the data in the data column to remove all the data, and get a new sequence of the percentage of the average. Let the original $n+1$ sequence be $\left\{x_{i}^{(0)}(k)\right\} \quad(i=0,1, \cdots, n ; k=1,2, \cdots, m)$. The average of the $i$ sequence is: 


$$
\overline{x_{i}}=\frac{\sum_{k=1}^{m} x_{i}^{(0)}(k)}{m}
$$

The new sequence after data averaging is:

$$
\left\{x_{i}^{(1)}(k)\right\}, \quad x_{i}^{(1)}(k)=\frac{x_{i}^{(0)}(k)}{\overline{x_{i}}}
$$

Step 2, Calculating the relational coefficient. The correlation coefficient is to consider the difference between the geometrical curves of the data columns, and the difference between the curves is used as the basis for measuring the correlation coefficient. And the correlation coefficient between $x_{i}$ and $x_{0}$ at point $k$ is:

$$
L_{0 i}(k)=\frac{\min _{i} \min _{k}\left|x_{0}^{1}(k)-x_{i}^{1}(k)\right|+\xi \max _{i} \max _{k}\left|x_{0}^{1}(k)-x_{i}^{1}(k)\right|}{\left|x_{0}^{1}(k)-x_{i}^{1}(k)\right|+\xi \max _{i} \max _{k}\left|x_{0}^{1}(k)-x_{i}^{1}(k)\right|}
$$

formula, $\min _{i} \min _{k}\left|x_{0}^{1}(k)-x_{i}^{1}(k)\right|$ is the minimum difference between the two poles; $\max _{i} \max _{k}\left|x_{0}^{1}(k)-x_{i}^{1}(k)\right|$ is the maximum difference between the two poles; order $\Delta_{0 i}(k)=\left|x_{0}^{1}(k)-x_{i}^{1}(k)\right|$, the above formula can be simplified as:

$$
L_{0 i}(k)=\frac{\min _{i} \min _{k} \Delta_{0 i}(k)+\xi \max _{i} \max _{k} \Delta_{0 i}(k)}{\Delta_{0 i}(k)+\xi \max _{i} \max _{k} \Delta_{0 i}(k)}
$$

in the formula, $\xi \in(0, \infty)$. it is called the resolution coefficient. The smaller the resolution, the larger the $\xi$ value range is $(0,1)$. The specific value depends on the situation. When $\xi \leq 0.5463$, the resolution was the best, $\xi=0.5$ usually taken.

Step 3, Calculating the relational degree and sorting the relational degree. The correlation coefficient is the correlation degree between the comparison sequence and the reference sequence at each moment (each point in the curve), the data is more, and the information is too scattered to facilitate the overall comparison. Therefore, it is necessary to concentrate the correlation coefficients at each time into one value, that is, to find the average value as a quantitative representation of the degree of association between the comparison sequence and the reference sequence. The calculation formula for the correlation $r_{0 i}$ between the comparison sequence $x_{0}$ and the reference sequence $x_{0}$ is:

$$
r_{0 i}=\frac{1}{m} \sum_{k=1}^{m} L_{0 i}(k)
$$

The degree of association between factors is mainly described by the order of the degree of relevance, not just the degree of relevance. The greater the degree of association, the closer the relationship between the reference sequence and the comparison sequence, the smaller the degree of association, the less closely the relationship between the reference sequence and the comparison sequence.

\section{Indicators on Disposable Income of Residents}

According to the source of income, Disposable income of residents contains four items, as shown in Fig.1.

(1) Wage income, refers to all the labor remuneration and various benefits that the employed person obtains through various means, including all labor remuneration and benefits obtained from the employer or individual, engaged in various freelance, part-time and sporadic labor.

(2) Net income of property, refers to the net income earned by households or household members 
engaged in production and business activities, which is the net income after deducting operating expenses, depreciation of productive fixed assets and production tax from all operating income.

(3) Net income of property, including net interest income, dividend income, net income from savings insurance, net income from transfer of contracted land management rights, net income from rental housing, net income from other assets, and net rental of self-owned housing. Net income from property does not include premium income from the transfer of ownership of the asset.

(4) Transferred net income refers to various recurring transfer payments to households by the state, units and social groups and recurring income transfers between households. Including old-age pension or retirement pension, social assistance and subsidies, policy production subsidies, policy-based living allowances, disaster relief payments, recurrent donations and compensation, reimbursement of medical expenses, and maintenance income between households. Back to income and so on.

\begin{tabular}{|c|c|}
\hline \multicolumn{2}{|c|}{ Disposable income of residents } \\
\hline Wage income & Net income of property \\
\hline Net operating income & Transferred net income \\
\hline
\end{tabular}

Fig. 1. Indicators on disposable income of residents

\section{Indicators on Consumption Expenditure of Residents}

The consumption of residents can be divided into eight kinds, as shown in Fig.2.

\begin{tabular}{|c|c|}
\multicolumn{2}{c|}{ Consumption expenditure of residents } \\
\hline Food, tobacco and alcohol & Traffic communication \\
\hline Clothing & Education, culture and entertainment \\
\hline Live & Health care \\
\hline Daily necessities and services & Other supplies and services \\
\hline
\end{tabular}

Fig. 2. Indicators on consumption expenditure of residents

(1) Food, tobacco and alcohol. Refers to the expenditure on various foods, tobacco and alcohol.

(2) Clothing. Refers to expenditures related to the wearings of residents, including clothing, clothing materials, footwear, other clothing and accessories, clothing and related processing services.

(3) Live, refers to expenditure related to residence, including expenses such as rent, water, electricity, fuel, property management, etc., and also includes rents for own houses.

(4) Daily necessities and services. Refers to various household, personal lifestyle and family services. Including furniture and upholstery, household appliances, home textiles, household groceries, personal items and family services.

(5) Traffic communication. Refers to transportation and communication tools and related various service fees, maintenance fees and vehicle insurance.

(6) Education, culture and entertainment. Refers to expenditures on education, culture and entertainment.

(7) Health care. Refers to the total cost of medicines, supplies, services for medical and health care. Includes medical equipment and medicines, as well as medical services.

(8) Other supplies and services. Other supplies and services that cannot be directly included in the above kinds of expenses. 


\section{Basic Data on Grey Relational Analysis}

The basic data studied in this paper comes from the 2017 China Statistical Yearbook of the National Bureau of Statistics of the People's Republic of China, as shown in Table 1. The indicators are expressed as follows: $x_{0}$, Disposable income of residents; $x_{1}$, Food, tobacco and alcohol; $x_{2}$, Clothing; $x_{3}$, Live; $x_{4}$, Daily necessities and services; $x_{5}$, Traffic communication; $x_{6}$, Education, culture and entertainment; $x_{7}$, Health care; $x_{8}$, Other supplies And services.

Table 1. Disposable income and consumption expenditure for urban residents in 2013-2016 years

\begin{tabular}{c|c|c|c|c|c|c|c|c|c}
\hline Year & $x_{0}$ & $x_{1}$ & $x_{2}$ & $x_{3}$ & $x_{4}$ & $x_{5}$ & $x_{6}$ & $x_{7}$ & $x_{8}$ \\
\hline 2013 & 18310.8 & 4126.7 & 1027.1 & 2998.5 & 806.5 & 1627.1 & 1397.7 & 912.1 & 324.7 \\
\hline 2014 & 20167.1 & 4493.9 & 1099.3 & 3200.5 & 889.7 & 1869.3 & 1535.9 & 1044.8 & 358.0 \\
\hline 2015 & 21966.2 & 4814.0 & 1164.1 & 3419.2 & 951.4 & 2086.9 & 1723.1 & 1164.5 & 389.2 \\
\hline 2016 & 23821.0 & 5151.0 & 1202.7 & 3746.4 & 1043.7 & 2337.8 & 1915.3 & 1307.5 & 406.3 \\
\hline
\end{tabular}

\section{Process on Grey Relational Analysis}

Step 1, Data normalization. The processing results are shown in Table 2.

Table 2. Data normalization processing results

\begin{tabular}{c|c|c|c|c|c|c|c|c|c}
\hline Year & $x_{0}$ & $x_{1}$ & $x_{2}$ & $x_{3}$ & $x_{4}$ & $x_{5}$ & $x_{6}$ & $x_{7}$ & $x_{8}$ \\
\hline 2013 & 0.8692 & 0.8881 & 0.9144 & 0.8974 & 0.8739 & 0.8217 & 0.8507 & 0.8238 & 0.8786 \\
\hline 2014 & 0.9573 & 0.9672 & 0.9786 & 0.9579 & 0.9641 & 0.9440 & 0.9348 & 0.9436 & 0.9687 \\
\hline 2015 & 1.0427 & 1.0361 & 1.0363 & 1.0234 & 1.0310 & 1.0538 & 1.0488 & 1.0517 & 1.0532 \\
\hline 2016 & 1.1308 & 1.1086 & 1.0707 & 1.1213 & 1.1310 & 1.1805 & 1.1657 & 1.1809 & 1.0994 \\
\hline
\end{tabular}

Step 2, Calculating the relational coefficient.

First, the Calculation of the minimum and maximum difference of the two stages, the results are shown in Table 3.

Table 3. Calculation of the minimum and maximum difference of the two stage

\begin{tabular}{c|c|c|c|c|c|c|c|c|c}
\hline $\begin{array}{c}\text { Classificati } \\
\text { on }\end{array}$ & $\begin{array}{c}\text { Final } \\
\text { value }\end{array}$ & $X_{0}-X_{1}$ & $X_{0}-X_{2}$ & $X_{0}-X_{3}$ & $X_{0}-X_{4}$ & $X_{0}-X_{5}$ & $X_{0}-X_{6}$ & $X_{0}-X_{7}$ & $X_{0}-X_{8}$ \\
\hline Minimum & 0.0002 & 0.0066 & 0.0064 & 0.0004 & 0.0002 & 0.0011 & 0.0061 & 0.009 & 0.0094 \\
\hline Maximum & 0.0601 & 0.0222 & 0.0601 & 0.0282 & 0.0117 & 0.0475 & 0.0185 & 0.0454 & 0.0114 \\
\hline
\end{tabular}

Then, Calculating the relational coefficient, the results are shown in Table 4.

Table 4. Calculation result of relational coefficient

\begin{tabular}{c|c|c|c|c|c|c|c|c}
\hline Year & $x_{1}$ & $x_{2}$ & $x_{3}$ & $x_{4}$ & $x_{5}$ & $x_{6}$ & $x_{7}$ & $x_{8}$ \\
\hline 2013 & 0.6180 & 0.4020 & 0.5193 & 0.8705 & 0.3901 & 0.6231 & 0.4009 & 0.7668 \\
\hline 2014 & 0.7572 & 0.5891 & 0.9869 & 0.8209 & 0.6978 & 0.5756 & 0.6914 & 0.7298 \\
\hline 2015 & 0.8254 & 0.8300 & 0.6130 & 0.7246 & 0.7351 & 0.8368 & 0.7746 & 0.7460 \\
\hdashline 2016 & 0.5789 & 0.3356 & 0.7649 & 1.0000 & 0.3793 & 0.4657 & 0.3774 & 0.4923 \\
\hline
\end{tabular}

Step 3, Calculating the relational degree and sorting the relational degree. The correlation degree result is as shown in the first row of the data in Table 5, and the correlation degree ranking result is as shown in the second row. 
Table 5. Calculation result of relational degree

\begin{tabular}{c|c|c|c|c|c|c|c|c}
\hline Calculation result & $x_{1}$ & $x_{2}$ & $x_{3}$ & $x_{4}$ & $x_{5}$ & $x_{6}$ & $x_{7}$ & $x_{8}$ \\
\hline Relational degree & 0.6949 & 0.5392 & 0.7210 & 0.8540 & 0.5506 & 0.6253 & 0.5611 & 0.6837 \\
\hline Sorting & 3 & 8 & 2 & 1 & 7 & 5 & 6 & 4 \\
\hline
\end{tabular}

\section{Analysis on Grey Relational Results}

According to the second row of Table 4 data. Disposable income of residents has a significant impact on the consumption indicators: Daily necessities and services $\left(x_{4}\right)$; Live $\left(x_{3}\right)$; Food, tobacco and alcohol $\left(x_{1}\right)$; other supplies and services $\left(x_{8}\right)$; Education, culture and entertainment $\left(x_{6}\right)$; Health care $\left(x_{7}\right)$; Traffic communication $\left(x_{5}\right)$; Clothing $\left(x_{2}\right)$. From the sort results can be derived:

(1) "Daily necessities and services" and "Disposable income of residents" have the greatest relevance, indicating that when "Disposable income of residents" increases, more household items are purchased and better living services are enjoyed.

(2) The relationship between "Food, tobacco and alcohol" and "Disposable income of residents" is also very large, indicating that when "Disposable income of residents" increases, the consumption expenditure on "Food, tobacco and alcohol" increases obviously.

(3) The degree of association between "Disposable income of residents" and "Clothing" is small, indicating that when the "Disposable income of residents" increases, the increase in consumer spending on "Clothing" is not obvious.

\section{Acknowledgement}

This work is supported by teaching reform project of Bohai University (BDJG2016QC06): Research and Practice on how to play the role of extracurricular classroom in higher mathematics teaching.

\section{References}

[1] Zhang Wei-qing, "Grey Associated Analytical on Rural Per Capita Net Income and Spending in China," Journal of Nanjing Institute of Industry Technology, vol. 11, no. 3, pp. 1-3, 2011.

[2] National Bureau of Statistics of the People's Republic of China, "China Statistical Yearbook 2017," http://www.stats.gov.cn/tjsj/ndsj/2017/indexch.htm, 2018-6-26.

[3] Baidu Wenku, "Grey relational analysis and its application cases," https://wenku.baidu.com/view/72ca9bf25901020207409cdf.html, 2018-6-26.

[4] H. T. Sun, "Co integration analysis of the relationship between disposable income and consumption expenditure of urban residents," Commercial Times, vol. 30, no. 30, pp. 26-28, 2011.

[5] L Wu, "Analysis on Relation between Income and Consumption Expenditures of Urban and Rural Residents in Henan Province," Journal of Jiyuan Vocational and Technical College, vol. 13, no. 4, pp. 65-68, 2014. 